Dom. Cien., ISSN: 2477-8818

Vol. 4, núm. 1, enero, 2018, pp. 280-289

Algunas consideraciones acerca del trastorno del aprendizaje

Ciencias de la educación

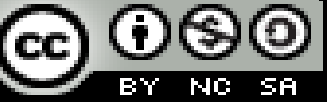

Artículo de investigación

\title{
Algunas consideraciones acerca del trastorno del aprendizaje
}

\author{
Some considerations about the learning disorder
}

\section{Algumas considerações sobre o transtorno de aprendizagem}

Recibido: 12 de agosto de $2017 *$ Corregido: 05 de noviembre de $2017 *$ Aceptado: 27 de diciembre de 2017

I Magister en Gerencia Educativa, Licenciada en Ciencias de la Educación especialidad Psicología y Orientación Vocacional, Docente de la Universidad Laica Eloy Alfaro de Manabí, Manta, Ecuador. 


\title{
Resumen
}

Cuando la inteligencia de los niños es promedio, pero el rendimiento en los tests que miden la lectura, las matemáticas o la expresión escrita, está por debajo del nivel esperado, por inteligencia, edad y escolaridad, estamos ante trastornos específicos del aprendizaje. A tales efectos se realizó una revisión bibliográfica acerca de la temática. Con la aplicación de medidas preventivas, educación para la salud y tratamiento específico, la integración social y escolar desde la etapa infantil estará normalizada al llegar a la adolescencia.

Palabras clave: aprendizaje; dificultades con el aprendizaje.

\begin{abstract}
When children's intelligence is average, but performance on tests that measure reading, mathematics or written expression is below the expected level, by intelligence, age and schooling, we are facing specific learning disorders. To this end, a bibliographical review was made on the subject. With the application of preventive measures, education for health and specific treatment, social and school integration from the childhood stage will be normalized upon reaching adolescence.
\end{abstract}

Keywords: learning; difficulties with learning.

\section{Resumo}

Quando a inteligência das crianças é média, mas o desempenho em testes que medem leitura, matemática ou expressão escrita está abaixo do nível esperado, por inteligência, idade e escolaridade, enfrentamos transtornos de aprendizagem específicos. Para este fim, foi feita uma revisão bibliográfica sobre o assunto. Com a aplicação de medidas preventivas, a educação para a saúde e tratamento específico, a integração social e escolar do estágio infantil será normalizada após a adolescência.

Palavras chave: aprendizagem; dificuldades em aprender.

\section{Introducción}

El aprendizaje implica la construcción progresiva de organizaciones cognitivas, que se van estructurando a través de intercambio con el medio ambiente y de la experiencia que el sujeto vive en 
dicho medio. Ello expresa que el hombre dotado de una estructura biológica, al entrar en contacto con situaciones diversas, está en capacidad de procesar toda esa información y estructurarla de manera tal, que se interioricen aquellos aspectos que han resultados significativos para él; es por ello que son de vital importancia los factores emocionales y sociales, lo cual implica tomar en cuenta la relación consigo mismo y con los otros, en función de sus intereses, valores, actitudes y creencias. (Fernández Pérez de Alejo G. 2015).

En tal sentido se asume, el aprendizaje como un proceso mediador basado en la interacción recíproca, entre aspectos biológicos, cognitivos, emocionales, ambientales y conductuales, relacionado con adquisición y uso de la información y el conocimiento, los cuales son interiorizados por el sujeto a través de experiencias significativas, contextuadas socio- culturalmente. (Fernández Pérez de Alejo G. 2015).

Cualquier alteración que esté presente en las condiciones internas del sujeto, en términos de su estructura neuro- biológica y psicológica, sin afectar su integridad cognitiva; así como interferencias en su realidad ambiental: familiar, social y comunitaria, pueden ocasionar dificultades en su proceso de aprendizaje. (Fernández Pérez de Alejo G. 2015).

Las discapacidades del aprendizaje abarcan todo un espectro de trastornos, que afectan la forma en que el cerebro procesa la información. Eso hace que el entender algunos conceptos resulte difícil. Este tipo de trastorno puede ser leve o grave. (¿Qué es un trastorno del aprendizaje?, 2016).

La mayoría de los niños con trastornos del aprendizaje, tienen una inteligencia normal o superior al promedio. Sin embargo, se les dificultan determinadas áreas de aprendizaje. Quizás les sea fácil reconocer las letras del alfabeto de manera individual, pero les resulte confuso agruparlas para leer. $\mathrm{O}$ tal vez no puedan solucionar un ejercicio de matemáticas simple o armar un rompecabezas. (¿Qué es un trastorno del aprendizaje?, 2016).

\section{Definición de trastorno del aprendizaje}

El trastorno del aprendizaje se define como una dificultad inesperada, específica y persistente para la adquisición de un aprendizaje pese a una instrucción convencional, nivel de inteligencia y oportunidades socioculturales adecuadas. Estos trastornos se incluyen dentro de los trastornos del 
neurodesarrollo e interfieren en el aprendizaje de habilidades académicas y/o sociales y a menudo coexisten con problemas de conducta, de estado de ánimo y/o de ansiedad. (Trastornos del aprendizaje. 2012).

Según Malaga Dieguez (2010), para poder definir lo que son los Trastornos del Aprendizaje (TA), hay que tener muy presente que cada individuo tiene un ritmo propio para asimilar los aprendizajes. En un aula cualquiera de niños sanos, dotados de una inteligencia dentro del rango de la normalidad, nos encontramos con distintos tipos de niños: brillantes, que aprenden y asimilan los conceptos rápidamente y con facilidad; "normales", que aprenden al ritmo esperado y con las dificultades típicas; niños a los que aprender y asimilar los conceptos, les supone una mayor dificultad que a los demás, pero que con un mínimo esfuerzo/tiempo suplementarios llegan a superarlos sin problemas, $\mathrm{y}$ finalmente están los niños con TA. Estos últimos son pacientes que presentan problemas persistentes.

La definición más utilizada de trastorno del aprendizaje es la incapacidad persistente, inesperada y específica para adquirir de forma eficiente determinadas habilidades académicas (ya sea lectura, escritura, cálculo, dibujo, etc.), y que ocurren a pesar de que el niño tenga una inteligencia normal, siga una escolarización adecuada y su entorno socio-cultural sea favorable. (Shaywitz SE1998), (Galaburda A, 2003).

Las dificultades del aprendizaje se presentan en la infancia, con más frecuencia de lo que se piensa pero por diverso motivos, se diagnostican en numerosas ocasiones tarde o no llegar a detectarse.

Es frecuente encontrar a padres de niños que acuden a consulta, por que el niño tiene problema en la escuela, y refieren que a ellos les había ocurrido lo mismo, peor que no pudieron o no supieron hacer nada, ni sus padres ni profesores, se manifiestan que no quiere que a sus hijos le pase lo mismo. (Mateo - Mateo R, López Guinea C. 2011).

\section{Trastorno de aprendizaje más comunes}

Discalculia: se trata de un trastorno de aprendizaje relacionado con las matemáticas. En concreto, el pequeño que lo sufre se topa con una seria dificultad, para poder comprender y realizar diferentes cálculos. (Málaga Diéguez1, J. Arias Álvarez. 2010). 
TDAH: bajo estas siglas se incluye otro trastorno del aprendizaje, que se da en llamar Trastorno de Déficit de Atención e Hiperactividad. Se manifiesta en el menor a través de síntomas, tales como un bajo rendimiento en el colegio, una dificultad notable para concentrarse o para estar quieto durante mucho tiempo e incluso problemas para poder relacionarse de manera normal con los padres, los profesores o los demás alumnos. (Málaga Diéguez1, J. Arias Álvarez. 2010).

Dislexia: esta es quizás la dificultad de aprendizaje más conocida y extendida. Como se sabrá, quienes la sufren, se topan con serios problemas a la hora de entender lo que leen, cambian el orden de las letras y palabras, no son capaces de distinguir letras como la b y la d. En otro tipo de dislexia, la dificultad radica en la demora en relacionar una palabra con su significado, lo que retarda la comprensión. (Málaga Diéguez1, J. Arias Álvarez. 2010).

Disgrafía: se trata de un trastorno relacionado con la escritura, ya que el pequeño que la tiene presenta serias dificultades para escribir correctamente. De ahí que cualquier palabra, frase o texto que proceda a plasmar de su mano en una hoja tenga un aspecto inclinado, deforme, irreconocible, imposible de descifrar. (Málaga Diéguez1, J. Arias Álvarez. 2010).

El síndrome de asperger: se le conoce como un autismo leve, en donde los niños presentan inteligencia promedio o superior al promedio, así como precocidad verbal, que les genera dificultades para interactuar en entornos sociales. (Málaga Diéguez1, J. Arias Álvarez. 2010).

Discapacidad de la memoria y el procesamiento auditivo: dificultad para comprender y recordar palabras o sonidos, porque la memoria no está almacenándolas ni descifrándolas de manera correcta. (Málaga Diéguez1, J. Arias Álvarez. 2010).

Trastorno del espectro autista: el autismo incluye una variedad síntomas, que van de leves a graves como capacidades sociales deficientes, hipersensibilidad a imágenes y sonidos y dificultades para adaptarse al cambio, principalmente. (Málaga Diéguez1, J. Arias Álvarez. 2010).

El primer paso para manejar adecuadamente estos problemas, es identificar las dificultades que atraviesa un niño en el proceso de aprendizaje, para obtener la ayuda que necesita para salir adelante.

Una vez realizada la valoración y conociendo sus deficiencias se aplica el tratamiento, que consiste en aprendizaje interactivo, mediante estimulación y ejercicios de: atención, concentración, memoria, 
agilidad mental, madurez motriz y relajación muscular progresiva. También se trabajara en técnicas métodos y hábitos de estudios así como en charlas de motivación escolar.

El tiempo que dure el tratamiento, dependerá de cómo vaya respondiendo y evolucionando el niño. Si cree que su hijo tiene algunos de los problemas anteriormente descritos, consulte inmediatamente con su médico.

\section{Los trastornos de aprendizaje y las diferencias socio culturales}

Los niños culturalmente deprimidos y que viven en pobreza son definidos en algunos países desarrollados como "minorías sub culturales", en comparación con la mayoría de alumnos que disponen de recursos económicos y culturales, que les han permitido lograr un desarrollo cognitivo cuando ingresan a la escuela y una motivación suficiente para el aprendizaje escolar. En países sudamericanos los niños que viven en pobreza constituyen una mayoría, y esa pobreza no sólo afecta a los alumnos y a sus familias, sino también a la calidad de sus escuelas. Como consecuencia, el rendimiento escolar es insuficiente y muchas veces su lentitud para aprender se confunde con trastornos específicos del aprendizaje o dislexias. (Bravo-Valdivieso, L et al 2009).

Algunas investigaciones comparativas muestran que las habilidades verbales y fonológicas entre niños de escuelas públicas gratuitas y de escuela privadas pagadas tienen un desfase inicial desde Kindergarten y primer año básico, antes que las escuelas puedan intervenir en superar las diferencias. (Bravo-Valdivieso, L et al 2009).

Las investigaciones en retardo lector y en dislexias en niños de bajo nivel sociocultural y económico, también presentan problemas metodológicos severos debido a sus diferencias psicolingüísticas y familiares. Muchos de esos niños tienen dificultad cultural para el reconocimiento fonémico y semántico de los textos escritos, letras, sílabas y palabras, que van produciendo un retraso acumulativo del aprendizaje del lenguaje escrito, que se manifiesta con claridad a finales de la educación básica. (Bravo-Valdivieso, L et al 2009).

Investigación realizada por Izaguirre Mejías (2012), referente a la intervención educativa en niños y niñas con dificultades especifica de aprendizaje, relacionada con el conocimiento y disposición del docente, demostró que tanto al variable conocimiento como la variable disposición docente, mostraron 
una correlación positiva que explica y predice con bastante aproximación las intervenciones educativas de las dificultades específicas de aprendizaje.

\section{Medidas preventivas}

El autor Magaña M (2014), plantea que la infancia y la adolescencia son las edades más receptivas para las intervenciones preventivas, especialmente para formar conciencia individual, así como de los conocimientos, capacidades y motivaciones para la adquisición de hábitos.

Conocer los factores de vulnerabilidad es imprescindible, para tomar medidas porque la prevención es fundamental tanto en la infancia como en la adolescencia.

Por tanto:

a. Disponer de medios humanos y técnicos para detección de los trastornos, como garantía para mejorar el rendimiento escolar.

b. Intervención prioritaria.

c. Coordinación y coherencia de programas y equipos.

d. Acción articulada de las instituciones implicadas: educación y salud.

e. Evaluación y coordinación de recursos escolares y sociales, para evitar la repetición de curso, que rara vez da buenos resultados.

f. Programas de educación para la salud integral y la prevención en los centros escolares.

g. Atención y adecuación individualizada de cada programa.

h. Información, educación y prevención son claves para conseguir un futuro saludable.

Para concluir, con la aplicación de medidas preventivas, educación para la salud y tratamiento específico, la integración social y escolar desde la etapa infantil estará normalizada al llegar a la adolescencia. 


\section{Referencia Bibliográficas}

Bravo-Valdivieso, $\mathrm{L}$ et al. Trastornos del aprendizaje: investigaciones psicológicas y psicopedagógicas en diversos países de sud américa. Cienc. Psicol. . 2009, vol.3, n.2, pp.203-218. ISSN 1688-4221. Disponible en: $<$ http://www.scielo.edu.uy/scielo.php?script=sci_arttext\&pid=S1688$42212009000200009 \& \operatorname{lng}=\mathrm{es} \& \mathrm{nrm}=\mathrm{iso}>$

Fernández Pérez De Alejo G .2015. Trastornos del aprendizaje o dificultades en el aprendizaje financieras [consulta 10 julio 2017]. Disponible en: www.sld.cu/.../pdf/.../trastornos_del_aprendizaje_o_dificultades_en_el_aprendizaje.p

Galaburda A, Cestnik L.2003. Dislexia del desarrollo. Rev Neurol; 36: S3-9

Izaguirre Mejías M S. 2012. La intervención educativa en niños y niñas con dificultades especifica de aprendizaje relacionada con el conocimiento y disposición del docente. Tesis para optar por el título de máster en formación de formadores de docentes de educación básica, [consulta 10 julio 2017]. Disponible en: www.cervantesvirtual.com/.../intervencion-educativa-en-ninos-y-ninas-condificultad

Málaga Diéguez1, J. Arias Álvarez 2. 2010. Los trastornos del aprendizaje. Definición de los distintos tipos y sus bases neurobiológicas. Boletín de la Sociedad de Pediatría de Asturias, Cantabria, Castilla y León. Vol. 50 n 211

Mateo - Mateo R, López Guinea C. 2011. Dificultades de aprendizaje. Revista educativa inclusiva. 4 (1). Disponible en www.ujaen.es/revista/rei/linked/documentos/documentos/12-7.pdf

Magaña M, Ruiz-Lázaro P. 2014. Trastornos específicos del aprendizaje. [Consulta 10 julio 2017]. Disponible en: https://www.sepeap.org/wp-content/.../Ps_inf_trastornos_especificos_aprendizaje.pdf

¿Qué es un trastorno del aprendizaje? [Sitio web].2016 [consulta 10 julio 2017]. Disponible en: https://espanol.babycenter.com/a11000098/qu\%C3\%A9-es-un-trastorno-del-aprendizaje 
Shaywitz Se. Dyslexia. N 1998. Engl J Med; 338: 307-12

Trastornos del aprendizaje. [Sitio web]. 2012 [consulta 10 julio 2017]. Disponible en: http://www.fundacionadana.org/definicion-y-tipos-trastornos-del-aprendizaje 